\title{
Evaluation of new Beckman Coulter 25(OH) Vitamin D assay and potential improvement of clinical interpretation
}

\author{
Özlem Ç. Madenci*1, Asuman Orçun', Zeynep Yildiz', Rana Sirmali', Nazan Tunçbilek², Nihal Yücel', Yusuf Temel', Aycan Bölük' \\ 'Biochemistry Laboratory, Dr. Lütfi Kırdar Kartal Research and Training Hospital, Istanbul, Turkey \\ ${ }^{2}$ Centro Laboratory, Istanbul, Turkey \\ *Corresponding author: ocmadenci@hotmail.com
}

\begin{abstract}
Introduction: The aim of this study was to assess the analytical performances of the newly developed Access2 25-hydroxyvitamin D (25(OH)D) total immunoassay on two analysers, Dx1800 and Access2 (Beckman Coulter, Brea, CA, USA), and compare these two and a recalibrated Modular E 170 25(OH)D assay (Roche Diagnostics, Penzberg, Germany) with reference liquid chromatography tandem-mass spectrometry (LC-MS/MS) with special emphasis on clinical diagnosis.
\end{abstract}

Materials and methods: Beckman immunoassays were assessed for imprecision, accuracy, limit of blank (LoB), limit of detection (LoD), limit of quantitation (LoQ), linearity, interference, and carryover. One hundred and nineteen samples were run on DxI 800, Access2, and E 170, and agreement with the LC-MS/MS method was evaluated.

Results: Dxl 800 and Access2 assays showed good performances in terms of LoB, LoD, LOQ, linearity, and interference. All immunoassays showed negative biases ranging from - 8.6\% (Dxl 800) to - 19.2\% (Access2). Dxl 800 and Access2 systems had proportional biases, and the E170 system had a constant bias with the largest random error. Concordance correlation coefficient values ranged from 0.941 (Cl: $0.917-0.958$ ) for Dxl800 to 0.854 (Cl: 0.811-0.889) for Access2. Kappa (K) coefficients were found moderate for DxI (0.709; Cl: 0.581-0.837) and E170 (0.771; Cl: 0.587-0.844) and fair for Access2 (0.572; Cl: 0.428-0.716).

Conclusions: All immunoassays can be used in routine $25(\mathrm{OH}) \mathrm{D}$ measurements, still fairly diagnosing patients' status. Recent standardization attempts seem not to contribute too much to clinical diagnosis. A clinical laboratory must at least be aware of its method to avoid misinterpretation of results.

Key words: vitamin D; method comparison; standardization; 25-Hydroxyvitamin D

\section{Introduction}

Vitamin D (VitD) deficiency results in abnormalities in bone metabolism such as rickets, osteomalacia, and osteoporosis. Moreover, it has been found to be related to a variety of non-bone-related conditions such as cancer, diabetes, and autoimmune and cardiovascular diseases $(1,2)$. This increased the demands for VitD testing and forced clinical laboratories to demand for accurate and simple methods suitable for routine measurements (3).

Immunoassays, liquid chromatography tandemmass spectrometry (LC-MS/MS), and high-performance liquid chromatography are the analytical techniques currently used for 25-hydroxyvitamin $\mathrm{D}(25(\mathrm{OH}) \mathrm{D})$ measurement (4). Ancient immunoassays had poor analytic performances in terms of accuracy, precision, linearity, and agreement with reference methods. Poor antibody specificity with cross-reactivity to other VitD metabolites, incomplete extraction of the $25(\mathrm{OH}) \mathrm{D}$ analyte from the vitamin $\mathrm{D}$ binding protein, and confounding matrix substances such as lipids are proposed as potential reasons for the significant differences in the $25(\mathrm{OH}) \mathrm{D}$ determination between various assays. Inter-assay variations caused confusion in clinical 
diagnosis. On the other hand, chromatography/ mass spectrometry-based assays are highly sensitive and specific for measuring serum 25(OH)D concentrations, but the high equipment costs and complex measurement procedures limit its widespread use. Thus, clinical laboratories largely rely on automated immunoassays with an improved accuracy and precision performance (5). To improve the quality of $25(\mathrm{OH}) \mathrm{D}$ testing, identification of a reference method and a reference standard has been debated in the last decade. An international effort to standardise the measurement of $25(\mathrm{OH}) \mathrm{D}$ and its metabolites is currently being led by the Vitamin D Standardization Program (VDSP), which was established in November 2010 by the National Institutes of Health Office of Dietary Supplements, Centres for Disease Control and Prevention, National Institute of Standards and Technology (NIST), and Ghent University (6). A NIST standard reference material (SRM 2972 and 972) has been proposed to improve the traceability and harmonization of 25(OH)D assays. Recently, new kits calibrated with reference material have been produced, or present ones are recalibrated to overcome inter-laboratory variability. The Access2 $25(\mathrm{OH}) \mathrm{D}$ assay is a competitive chemiluminescence enzyme immune assay, and Roche $25(\mathrm{OH}) \mathrm{D}$ total assay is a competitive electrochemiluminescence immunoassay, both presented as traceable to a new NIST standard.

The hypothesis of this study was that these newly calibrated immunoassays minimize inter-laboratory variations with an improvement in clinical decision. In this study, we aimed to assess the analytical performances of newly developed Access2 25(OH)D assay on two analysers, Dxl 800 and Access2 (Beckman Coulter, Brea, CA, USA), and compare these two and a recalibrated Roche $25(\mathrm{OH}) \mathrm{D}$ (Roche Diagnostics, Penzberg, Germany) assay with reference LC-MS/MS and see the improvement on clinical decision.

\section{Materials and methods}

\section{Study design and subjects}

This is an analytical method evaluation study; records of outpatients attending our institute on two consecutive days were evaluated. Adult patients (> 18 years) with no pathologic laboratory results and taking no medications were selected. A total of 124 samples out of remnant sera were included in the study. After LC-MS/MS measurements, 4 samples having measurable 25-hydroxyergocalciferol $(25(\mathrm{OH}) \mathrm{D} 2)$ concentrations were excluded because the number was insufficient to state a subgroup. One sample over the measuring range of E170 system was also excluded. The remaining 119 patient samples, with mean age of 58 years (range $18-80$ years) (54 females (45\%) and 65 males (55\%)) and with different concentrations of 25(OH)D (range 3.99-178.20 nmol/L), were evaluated.

\section{Blood sampling}

Blood sampling was performed after an overnight fasting at 8:00-10:00 am from the antecubital vein into $5 \mathrm{ml}$ BD Vacutainer Serum Separating Tubes II Advance Tube (Lot 6197520) (Becton, Dickinson and Company, BD Plymouth PL6 7BP, UK). Blood samples were centrifuged at $2000 \times \mathrm{g}$ for $10 \mathrm{~min}$ utes. Samples were divided in to four aliquots and stored at $-20^{\circ} \mathrm{C}$ for a maximum of 15 days and analysed in batches on four systems. Analytical performance of newly developed 25(OH)D assays was assessed on two platforms: UniCel Dxl 800 and Access2. The limit of blank (LoB), limit of detection (LoD), limit of quantitation (LoQ), linearity, interference, and carryover studies were performed on two Beckman platforms. Another 25(OH)D assay on E 170 (Roche Diagnostics, Penzberg, Germany) was included in precision, accuracy, and method comparisons since acceptable performance criteria were based on accuracy and imprecision. Other analytic performance characteristics of Roche were supplied by the manufacturer and of LC-MS/ MS were provided from the reference laboratory.

All studies were done according to the Clinical \& Laboratory Standards Institute (CLSI) Evaluation Protocols (EP) specific to each parameter.

LC-MS/MS measurements were performed at Centro Laboratories, a certified clinical laboratory in Istanbul. Three immunoassay measurements were performed in the biochemistry laboratory of Dr. 
Lütfi Kırdar Kartal Research and Training Hospital between March and May 2016. This study was approved by the Ethical Committee of our institution.

\section{Methods}

Special applications of Access2 25(OH)D total assay were designed for both Dxl 800 (Cat. No. A98856) and Access2 (Cat. No. B24838). The total coefficient of variations (CV) provided by the manufacturer were $9.3 \%$ for $38.94 \mathrm{nmol} / \mathrm{L}$ and $5.6 \%$ for $399 \mathrm{nmol} / \mathrm{L}$ for Dxl 800 and $7.5 \%$ for $61.4 \mathrm{nmol} / \mathrm{L}$ and $6.1 \%$ for $353 \mathrm{nmol} / \mathrm{L}$ for Access 2 . Linearity was given as 4.99-524 nmol/L for Dxl 800 and 4.99-416 $\mathrm{nmol} / \mathrm{L}$ for Access2. CV values for Roche 25(OH)D assay (Cat. No. 05894913) provided by the manufacturer were $6.8 \%$ for $20.4 \mathrm{nmol} / \mathrm{L}$ and $3.7 \%$ for $174 \mathrm{nmo} / \mathrm{L}$. Linearity was given as 7.5-175 nmol/L. Both Access 2 and Roche assays were claimed to measure both 25(OH)D2 and 25-hydroxycholecalciferol $(25(\mathrm{OH}) \mathrm{D} 3)$ as a total and traceable to the NIST reference material.

LC-MS/MS reference measurements were done with an in-house method in Centro Laboratories, a certified clinical laboratory in Istanbul. The analyzer was Triple Quad 4500 (AB SCIEX, Framingham, USA). Phenomenex Kinetex 2.6 um C8 100 An LC column was used. It quantifies $25(\mathrm{OH}) \mathrm{D} 3$ and 25(OH)D2 separately using atmospheric pressure chemical ionization and a deuterated internal standard. The system was calibrated with 6PLUS1 $25(\mathrm{OH}) \mathrm{D} 3 / 25(\mathrm{OH}) \mathrm{D} 2$ multilevel serum calibrator $(0$, 13, 39, 76, 152, 262, and $357 \mathrm{nmol} / \mathrm{L}$ ) (ref. no 62039, Chrome systems, Munich, Germany) traceable to the NIST 972 reference material. The method was linear in the concentration range of 9.98-374.4 $\mathrm{nmol} / \mathrm{L}$. The inter-assay CVs were $5.5 \%$ for 43.5 $\mathrm{nmol} / \mathrm{L}$ and $4.2 \%$ for $95.5 \mathrm{nmol} / \mathrm{L}$. This laboratory participated in Vitamin D External Quality Assessment Scheme (DEQAS). Previous five monthly external control results of this system showed biases of $-4.6 \%,-0.8 \%, 9.4 \%,-1.1 \%$, and $-2.7 \%$ from the target value, and peer group CVs were $10.9 \%$, $9.9 \%, 12.6 \%, 10.7 \%$, and $11.1 \%$, respectively.

\section{Assay performance studies}

\section{Imprecision}

Imprecision (within-run, between-run, and between-day) was determined using serum pools of two different $25(\mathrm{OH}) \mathrm{D}$ concentrations.

Imprecision (CV total) was determined using two serum pools at two different concentrations, low (32.2 nmol/L) and high (109.5 nmol/L), based on patient test results measured in our laboratory. According to the CLSI Guidelines for testing precision (EP5A), they were tested twice daily in duplicate, with a minimum of 4 hours between each run for 20 days. Total CVs were calculated for three immunoassay systems. Acceptable imprecision criteria was $\mathrm{CV} \leq 10 \%$ (7).

\section{Accuracy}

Three samples provided from Randox International Quality Assessment Scheme (RIQAS) external quality assessment monthly immunoassay program (code RQ9130) were used for evaluating accuracy. The first, second, and third samples of Cycle 14 were analyzed once with all the methods. Percent difference from the published target mean was determined as follows: ((result - mean) / mean) $\times 100$. The acceptable accuracy was defined as $30.2 \%$ for RIQAS.

\section{$L O B, L O D$, and $L O Q$}

Studies were done according to CLSI EP17 (8). LoB was determined by analyzing 20 replicates of manufacturer's zero calibrator and was calculated using the following formula:

$$
\text { LoB }=\text { Mean (blank) + 1.645 SD (blank). }
$$

The limit of detection was determined using the lowest non-zero calibrator (14.976 nmol/L) which was diluted (1/2), and 20 replicates were analysed. LoD was calculated using the following formula:

LoD $=$ LoB + 1.645 (SD low-concentration sample).

Limit of quantification study was performed by measuring samples with concentrations ranging from 4.822 to $38.281 \mathrm{nmol} / \mathrm{L}$ for 10 days. Six samples around the limit of quantitation indicated by 
the manufacturer $(14.976 \mathrm{nmol} / \mathrm{L})$ were measured, and CVs were determined. LoQ is the point at which the fitted curve crosses the $20 \%$ CV line.

\section{Linearity}

Linearity was assessed according to CLSI EP6 (9). By diluting the highest standard of each reagent kit, six different concentrations in the range of $23.5-525 \mathrm{nmol} / \mathrm{L}$ for $\mathrm{Dxl}$ and in the range of $17.5-$ $412 \mathrm{nmol} / \mathrm{L}$ for Access 2 were performed and analysed for three replicates in a single run. Acceptable recovery criteria were $\pm 15 \%$ from the target concentration.

\section{Interference studies}

Interference was tested for haemoglobin, bilirubin, and triglycerides according to CLSI EP7 A (10). All spiked samples were tested twice. The percent difference for each interferent studied was calculated using the average from the duplicate measurements ((spiked - nonspiked) / nonspiked) $\mathrm{x}$ 100). A deviation of more than $10 \%$ was considered significant.

For haemoglobin interference study, $6 \mathrm{~mL}$ of venous blood samples of healthy volunteers were collected to Plastic Whole Blood tubes spraycoated with $\mathrm{K}_{2}$ EDTA (BD Vacutainer, lot 367863) and centrifuged at $5000 \times \mathrm{g}$ for 5 minutes. Plasma was omitted, and the cell pocket at the bottom was washed three times with $8 \mathrm{~mL}$ of physiologic serum. Supernatant was omitted, and equal volume of distilled water was added on the erythrocyte pocket. The tube was placed in $-40^{\circ} \mathrm{C}$ for 20 minutes to get erythrocytes haemolysed. After last centrifugation hemolysate was obtained as supernatant. Haemoglobin concentration was measured at LH750 haematology analyzer (Beckman Coulter, Brea, CA, USA). Eight different concentrations of haemoglobin (range $0.25-42 \mathrm{~g} / \mathrm{L}$ ) was spiked into patient serum pools.

Bilirubin standard ( $\geq 98 \%$, Sigma Aldrich B4126, $\mathrm{EmM} / 453=60$ ) was dissolved in chloroform Merck (M102445.2500), and different bilirubin concentrations (range, 54.72-513 $\mu \mathrm{mol} / \mathrm{L}$ ) were spiked into serum pools for the determination of bilirubin interference.
Samples prepared from the lipid standard Intralipid 20\% (Sigma Aldrich) with four different triglyceride concentrations (7.67-21.8 $\mathrm{mmol} / \mathrm{L})$ were spiked into serum pools for the determination of triglyceride interference.

\section{Carryover}

Sample carryover was evaluated by measuring three replicates of a high-concentration sample (samples a1, a2, and a3) immediately followed by three replicates of a low-concentration sample (samples b1, b2, b3). Carryover was calculated using the equation (b1-b3) / (a3-b3), < 2\% was accepted as negligible $(11,12)$.

\section{Method comparison}

25(OH)D analyte concentrations of the samples within the measurement range of all systems were processed in a single batch, in duplicate, within the same freeze / thaw cycles. Method comparison studies were performed according to CLSI EP9 (13).

\section{Statistical Analysis}

The distribution of data were assessed by the Kolmogorov-Smirnov test, and results were expressed as median and interquartile range. EP Evaluator Release 9 software (David G Rhoads Association, Kennett Square, PA) was used to calculate imprecision, LoB, LoD, LoQ, and linearity. Method comparison data were evaluated using the Bland-Altman plots, Passing-Bablok regression, and concordance correlation coefficient (CCC), and kappa ( $\mathrm{k}$ ) coefficients were done with MedCalc Statistical Software (version 12, MedCalcSoftware, Mariakerke, Belgium). Systematic error was considered significant if the $95 \%$ confidence intervals did not include 1.0 for slope (proportional error) or 0 for the $y$-intercept (constant error).

Diagnostic accuracy was tested with the $\mathrm{k}$ coefficient. Taking $74.88 \mathrm{nmol} / \mathrm{L}$ as cut-off, patients were grouped as deficient and non-deficient according to LC-MS/MS results. Agreement of the test method in identifying patients was expressed with the $\mathrm{K}$ coefficient. The interpretation of $\mathrm{K}$ is as follows: 
0.41-0.60 moderate; $0.61-0.80$ substantial; and 0.81-0.99 almost in perfect agreement. Kappa should be greater than 0.61 to be considered acceptable (14).

\section{Results}

The assay performance studies are given in Table 1. The median (2.5-97.5 percentiles; $\mathrm{nmol} / \mathrm{L}$ ) values of 119 samples were 50.4 (12.7-161.9) for Access2, 56.3 (14.5-174) for Dxl 800, 59.7 (7.4-163) for E170, and 67.8 (13.8-174) for LC-MS/MS. All three systems deviated negatively from reference results. Access 2 with the smallest mean had a $22.2 \%$ deviation, while E170 had $11 \%$ and Dxl 800 had $10 \%$ deviations from LC-MS/MS. Box and whisker plots show the distribution of results for the four methods in Figure 1.

The Bland-Altman analysis yielded negative biases for all three immunoassay systems compared with the reference. All three biases were significantly different from zero $(P<0.05)$. However, none of them were $<5 \%$ as VDSP suggested. Dxl 800 had the smallest bias, $-8.6 \%$, and Access 2 had the largest at $-19.2 \%(P<0.001)$. The E170 system

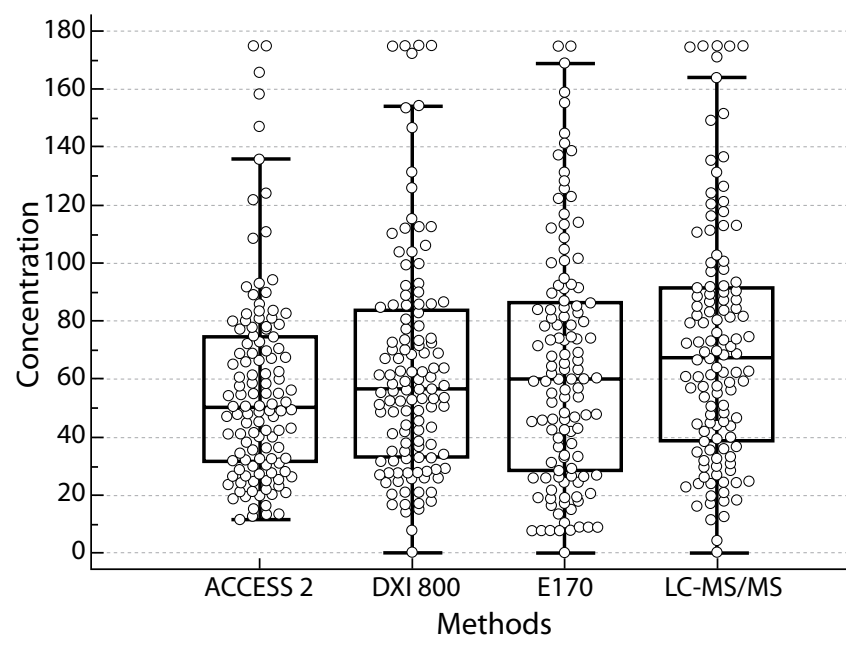

Figure 1. Box and whisker plots of 4 methods (Access2, Dxl 800, E170 and LC-MS/MS) for 25(OH)D measurements.

Median values for Access2, DXL 800, E170 and LC-MS/MS were $50.44,56.36,59.77$ and $67.39 \mathrm{nmol} / \mathrm{L}$, respectively, $\mathrm{P}=0.04$. Each box shows 25 to 75 percentile range. Horizontal line in each box represents group median.

had the largest limits of agreement (- 40.1-64.5\%), which is a measure of imprecision. Bland-Altman plots are shown in Figure 2.

TABLE 1. Performance characteristics of methods for 25(OH)D measurements

\begin{tabular}{|c|c|c|c|c|c|}
\hline Performance criteria & & $\begin{array}{c}\text { DXL } 800 \\
\text { System }\end{array}$ & $\begin{array}{l}\text { Access2 } \\
\text { System }\end{array}$ & $\begin{array}{c}\text { E } 170 \\
\text { System }\end{array}$ & $\begin{array}{l}\text { LC-MS/MS } \\
\text { System }\end{array}$ \\
\hline \multirow{2}{*}{$\begin{array}{l}\text { İmprecision } \\
\text { (Total CV\%) }\end{array}$} & Low & 8.9 & 8.1 & 5.2 & 3.9 \\
\hline & High & 8.2 & 7.7 & 2.2 & 1.7 \\
\hline \multirow{3}{*}{$\begin{array}{l}\text { Accuracy } \\
\text { (Deviation \%) }\end{array}$} & RIQAS 1 & -23.82 & -23.49 & 1.46 & 30.12 \\
\hline & RIQAS 2 & -1.08 & -7.13 & 5.88 & 62.82 \\
\hline & RIQAS 3 & -16.30 & 0.11 & 7.78 & 29.60 \\
\hline LoB (nmol/L) & & 2.48 & 1.92 & $4.99^{\dagger}$ & Unknown \\
\hline LoD (nmol/L) & - & 4.64 & 5.67 & $7.49^{\dagger}$ & 1.60 \\
\hline LoQ (nmol/L) & & 8.61 & 8.84 & $12.48^{\dagger}$ & 5.37 \\
\hline \multirow[t]{2}{*}{ Linearity (nmol/L) } & - & $23.5-525$ & $17.5-412$ & $7.5-175^{+}$ & $5.3-349$ \\
\hline & Hemoglobin $(\mathrm{g} / \mathrm{L})$ & $>0.50$ & $>0.50$ & $0.20^{\dagger}$ & * \\
\hline \multirow[t]{2}{*}{ Interference } & Triglyceride (mmol/L) & $>7.67^{\ddagger}$ & $>7.67^{\ddagger}$ & $>4.52^{\dagger}$ & * \\
\hline & Bilirubin (umol/L) & $>513$ & $>513$ & $>1129^{\dagger}$ & * \\
\hline Carry-over \% & - & 0.276 & 0.001 & Not identified $^{\dagger}$ & - \\
\hline
\end{tabular}

*No interference. ${ }^{\dagger}$ Values claimed by the manufacturer. ${ }^{\ddagger}$ Manufacturer claims were not met (Manufacturer claim was 37.06 $\mathrm{mmol} / \mathrm{L}$ ). RIQAS - Randox External Quality Assessment (EQA)/ Proficiency Testing (PT) scheme. LoB - Limit of blank. LoD - limit of detection. LoQ - limit of quantification. 

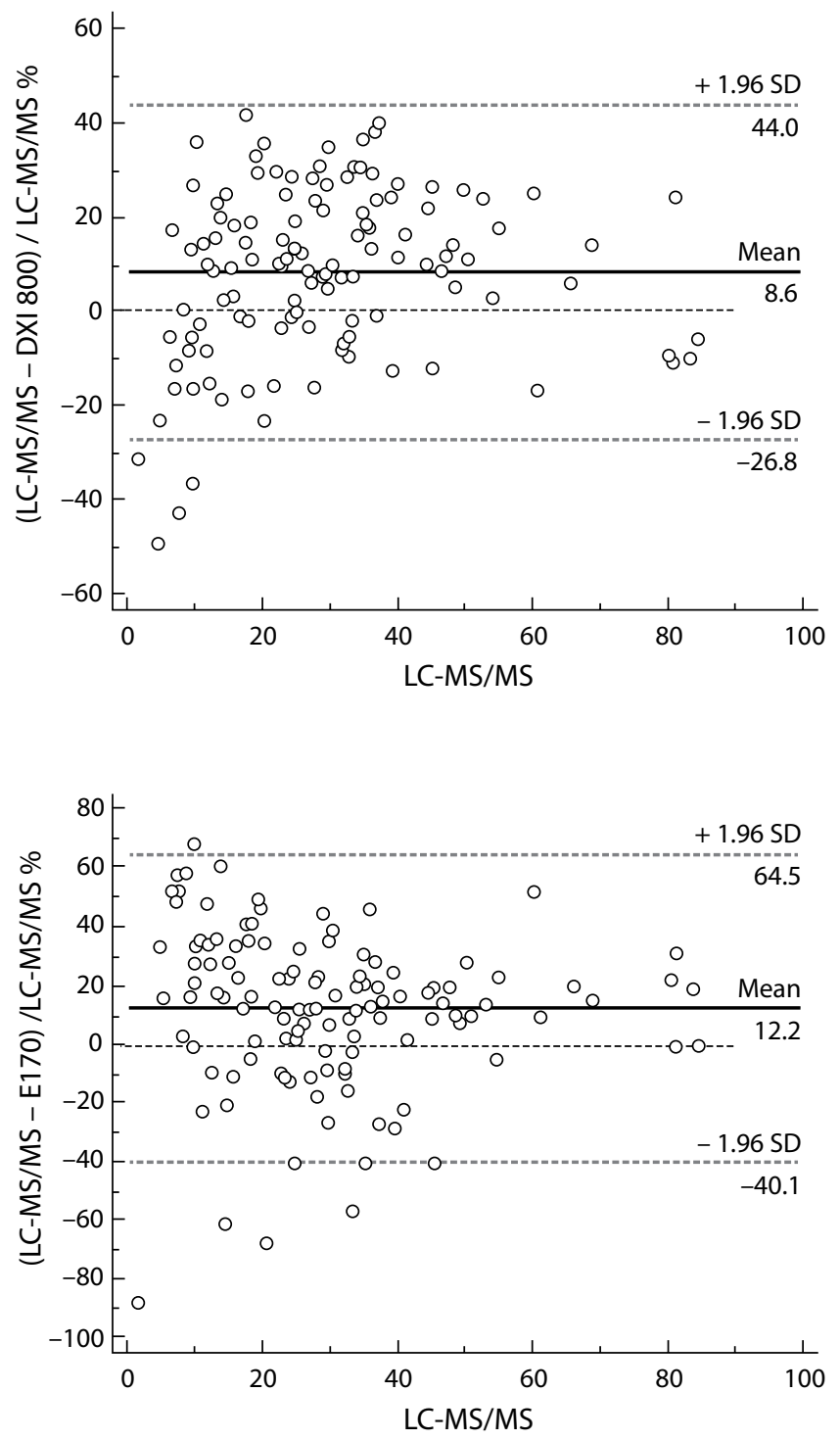

According to the Passing-Bablok regression analysis, the DxI 800 and Access2 systems had proportional biases (with slope values of 0.878 and 0.748 , respectively), while the E170 system had a constant bias with an intercept value of - 2.797. This system had the largest random error (residual standard deviation, 5.10) Passing-Bablok regression analysis is shown in Figure 3.

In concordance correlation analysis, the DxI 800 and E170 systems showed moderate agreement (CCC $=0.941$ and 0.901, respectively).

Kappa coefficients of interrater agreement were found to be moderate for DxI 800 and E170 ( $\mathrm{K}=$ 0.709 and 0.771 , respectively) and fair ( $\mathrm{k}=0.572$ ) for Access2 systems.

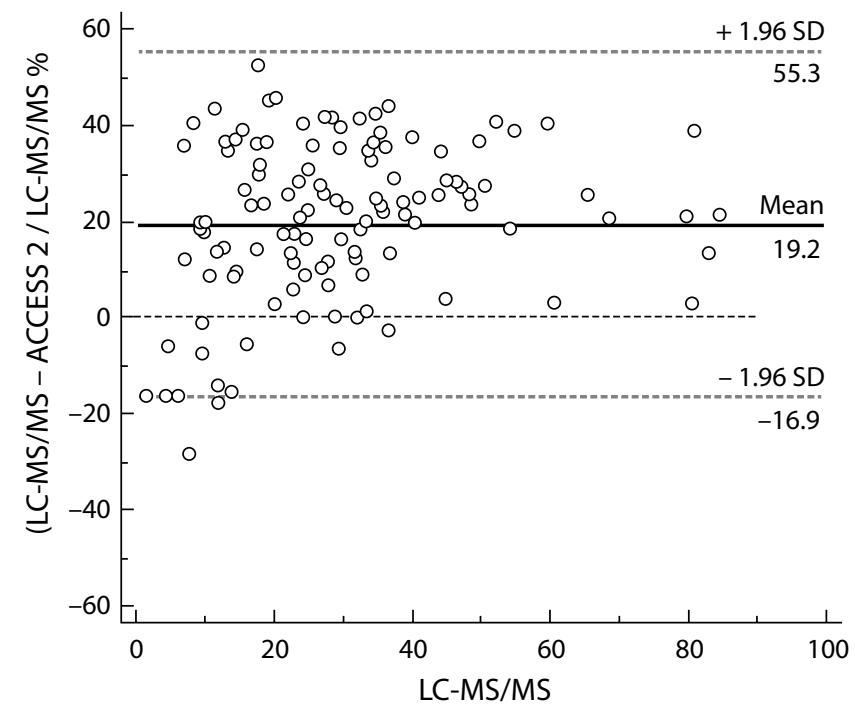

Figure 2. Bland-Altman plots of method comparison for $25(\mathrm{OH})$ D measurements.

Mean (thick solid line) - percentage bias (means of paired differences). Dashed lines demonstrate the $95 \%$ limits of agreement (bias \pm 1.96 standard deviation). Thin dotted line - line of equality.

Compared with Access2, the Dxl 800 system showed a positive bias of $12.7 \%(P<0.001)$ with an $R$ value of 0.95 (intercept 2.26 [Cl, 1.96-2.55], slope $0.78[\mathrm{Cl}, 0.74-0.82], \mathrm{P}<0.001)$ in regression analysis. Method comparison data are shown in Table 2.

\section{Discussion}

Access2 25(OH)D assay showed good performance in LoB, LoD, LoQ, linearity, interference, and carryover studies on both platforms Dxl 800 and Access2. Imprecision values for both low and high concentrations of 25(OH)D were acceptable $(<$ $10 \%)$. Accuracy was found acceptable for both systems based on RIQAS criteria (30.2\%). Method 

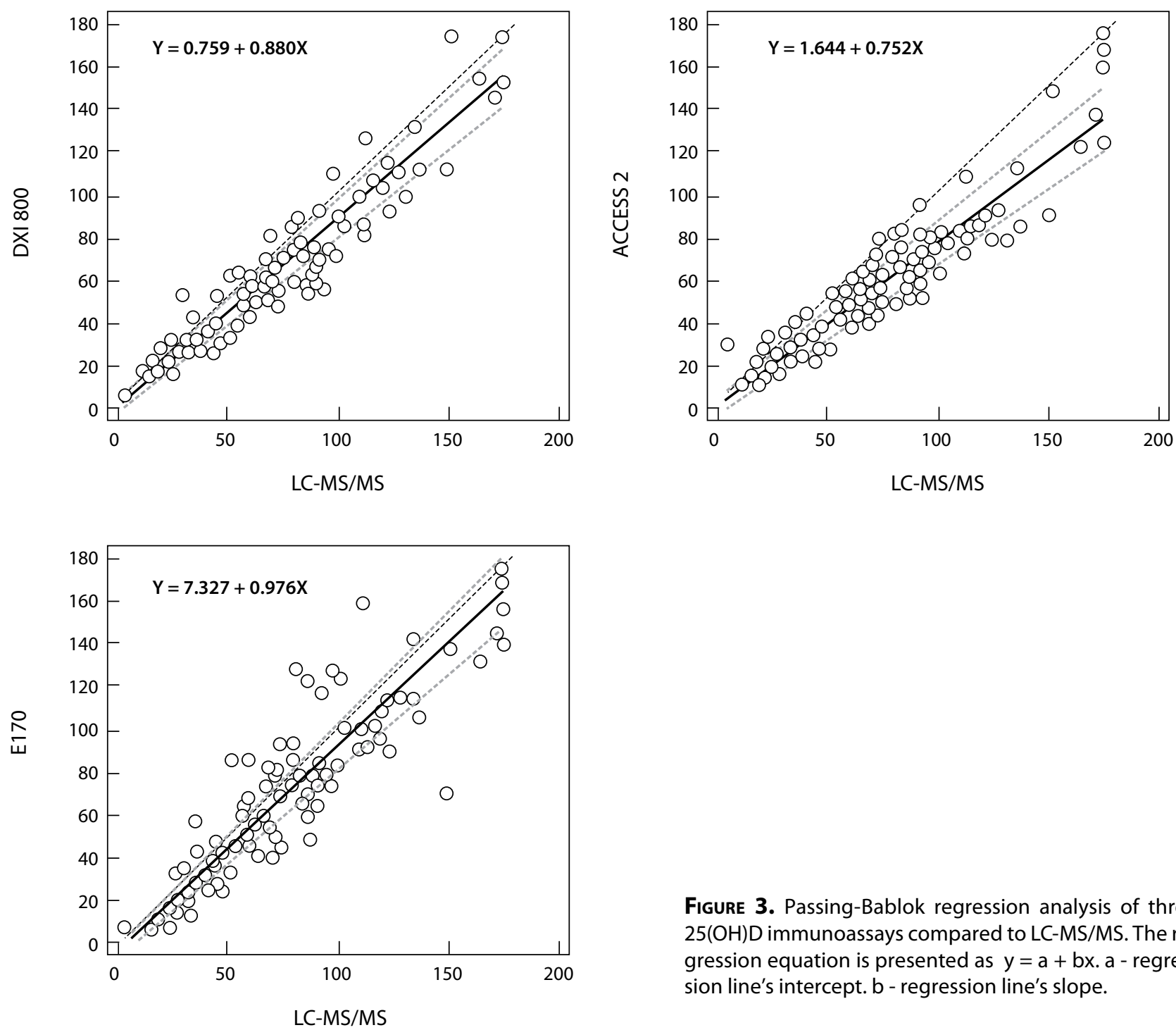

Figure 3. Passing-Bablok regression analysis of three $25(\mathrm{OH}) \mathrm{D}$ immunoassays compared to LC-MS/MS. The regression equation is presented as $y=a+b x$. $a$ - regression line's intercept. $b$ - regression line's slope.

TABLE 2. Method comparison results of immunoassays for $25(\mathrm{OH}) \mathrm{D}$ measurements with LC-MS/MS

\begin{tabular}{lccccccc}
\hline \multirow{2}{*}{ Method } & \multicolumn{2}{c}{$\begin{array}{c}\text { Passing-Bablok } \\
\text { regression analysis }\end{array}$} & \multicolumn{2}{c}{$\begin{array}{c}\text { Concordance correlation } \\
\text { analysis }\end{array}$} & \multicolumn{2}{c}{$\begin{array}{c}\text { Bland-Altman } \\
\text { analysis }\end{array}$} & $\begin{array}{c}\text { Interrater } \\
\text { aggreement }\end{array}$ \\
\cline { 2 - 7 } Dxl 800 & Slope $(\mathrm{Cl})$ & Intercept $(\mathrm{Cl})$ & $\mathrm{CCC}(\mathrm{Cl})$ & $\mathrm{P}$ & $\mathrm{C}_{\mathrm{b}}$ & Bias (\%) & $\mathrm{Kappa}(\mathrm{Cl})$ \\
\hline \multirow{2}{*}{ Access2 } & $\begin{array}{c}0.878 \\
(0.819-0.949)^{*}\end{array}$ & $\begin{array}{c}0.420 \\
(-1.120-1.762)\end{array}$ & $\begin{array}{c}0.941 \\
(0.917-0.958)\end{array}$ & 0.955 & 0.986 & -8.6 & 0.71 \\
\hline \multirow{2}{*}{ E170 } & 0.748 & 0.697 & 0.854 & 0.950 & 0.899 & -19.2 & $(0.43-0.72)$ \\
\hline
\end{tabular}

$\mathrm{Cl}$ - confidence interval. *Slope Cl's don't include 1.0 meaning proportional error. 'intercept Cl's don't include 0 meaning constant error. $C_{b}$ - Bias correction factor (accuracy). CCC - Concordance correlation coefficient. P - Pearson correlation coefficient (precision). 
comparison studies showed a persistent negative bias in all three immunoassays compared with LCMS/MS; the Dxl 800 system had the smallest bias (- 8.6\%), and Access2 had the greatest (- 19.2\%). The DxI 800 and E170 systems showed moderate agreement (CCC $=0.941$ and 0.901 , respectively), while Access 2 system was fair $(C C C=0.854)$. Taking $74.88 \mathrm{nmol} / \mathrm{L}$ as the cut-off for diagnostic insufficiency, DxI 800 and E170 systems differentiated insufficient patients moderately ( $\mathrm{K}$ values 0.709 and 0.771 , respectively) as Access 2 system did fairly $(k=0.572)$. Compared with Access 2 , the Dxl 800 system showed a positive bias of $12.7 \%(P<0.001)$ with an $R$ value of 0.95 (intercept $2.26[\mathrm{Cl}, 1.96-$ 2.55], slope 0.78 [Cl, 0.74-0.82], $\mathrm{P}<0.001)$.

There were significant biases and poor CVs in many of the previous studies about VitD (15-17). Even VDSP recommendations did not contribute too much to assay performances and clinical diagnosis (18-20). In these studies, the lack of definite acceptable performance criteria was an important issue. Different CVs, biases, and accuracy goals were set in different studies. Enko et al. used a CV $<20 \%$, referring to an opinion of the U.S. Department of Health and Human Services, Food and Drug Administration $(19,21)$. This study did not demonstrate a priori goal for bias. Farrell et al. used $\mathrm{CV}<9.1 \%$ and bias $<15.8 \%$ as performance goals based on biological variation studies $(16,22,23)$. Yu Chen used the DEQAS expert opinion criteria of $\mathrm{CV}<22 \%$ and bias $<10 \%$ and laboratory data model goals of $\mathrm{CV}<15 \%$ and bias $<10 \%$ as proposed by Stockl et al. $(12,24)$. Wyness et al. also used CV $<10 \%$ and bias $<15.8 \%$ as criteria based on the biologic variation saying that to establish a performance of $<5 \%$ for bias could hardly be attained (25). In our study, total CV and bias values of all assays were acceptable according to these established criteria in literature, but none of them achieved a bias of <5\%; Dxl 800 could be said to attain a comparable one (8.61\%).

LC-MS/MS is a precise and reliable method with high sensitivity and specificity and is also able to measure 25(OH)D2 and 25(OH)D3 separately. In the past, LC-MS/MS methods were not in agreement by themselves, and in a recent study, routine LC-MS/MS measurements were $11.2 \%$ higher than the standard reference procedure $(26,27)$. VDSP also identified a standard reference procedure for this method and expects routine laboratory LCMS/MS results to be traceable to this reference procedure by the use of NIST reference materials. In our study, the results of LC-MS/MS were significantly higher than all of the immunoassays. In accuracy-based studies we held with RIQAS external quality assurance samples, almost all immunoassays gave negative biases of up to - $23.82 \%$ (except one bias of $0.11 \%)$. When the same samples were measured with LC-MS/MS, it gave positive biases in the range of $29.6-62.82 \%$. Obviously, LC-MS/MS results were higher than immunoassays. Immunoassays were participants of the RIQAS, and the LCMS/MS system was a participant of the DEQAS Programme. The acceptable accuracy was defined as $25 \%$ for DEQAS and $30.2 \%$ for RIQAS (26). Thus, each method showed a comparable accuracy in its own program. The previous five biases obtained by LC-MS/MS in DEQAS were $-4.6 \%,-0.8 \%, 9.4 \%$, $1.1 \%$, and $-2.7 \%$, and the observed peer group CVs were $10.9 \%, 9.9 \%, 12.6 \%, 10.7 \%$, and $11.1 \%$ respectively. Regarding these data, Centro laboratory results had high accuracy, with peer group CVs showing the scatter of LC-MS/MS results seemingly higher than expected. Though some reports mentioned about recently improved agreement among LC-MS/MS methods (28), the CV values above reflect the variations in DEQAS participants.

Different from previous studies, we provided essential data describing both analytical and diagnostic performances of the Beckmann assays besides imprecision and accuracy performances of the new Roche assay. We saw that achieving analytical goals did not mean better clinical diagnosis. For instance, the Dxl 800 system showed an acceptable performance in terms of imprecision, accuracy, and bias, and it could moderately differentiate insufficient patients ( $\mathrm{K}=0.709)$ in agreement statistics. From the point of clinical practice, these numerical data mean a misdiagnosis of 33\% (16/49) of nondeficient patients as deficient. Thus, we say that analytical and clinical aspects of a method performance should always be considered together.

An important limitation of this study was the lack of a subgroup containing VitD2. The majority of 
supplements mostly used do not contain VitD2 in Turkey; thus, we could only detect measurable amounts of 25(OH)D2 in four patients' sera and excluded them. Moreover, 3-epi-25(OH)D3, which might be present in sera of children (29), was not measured in this study. To avoid the influence of this epimer, we did not include children's sera in the study.

\section{References}

1. Rosen CJ, Adams JS, Bikle DD, Black DM, Demay MB, Manson JE, et al. The nonskeletal effects of vitamin D: An Endocrine Society scientific statement. Endocr Rev 2012;33:456-92. https://doi.org/10.1210/er.2012-1000

2. Holick MF. Vitamin D deficiency. NEngl J Med 2007;357:26681. https://doi.org/10.1056/NEJMra070553

3. Holick MF. Vitamin D status: measurement, interpretation, and clinical application. Ann Epidemiol 2009;19:73-8. https://doi.org/10.1016/j.annepidem.2007.12.001

4. Ozcan N, Ucar F, Arzuhal AE, Bulut E, Ozturk A, Yavuz Taslipinar $M$, et al. Evaluation of the analytical performance of Unicel DXL 800 for the Total $25(\mathrm{OH})$ Vitamin D measurements. Clin Biochem 2016;49:486-91. https://doi. org/10.1016/j.clinbiochem.2015.11.022

5. Lai JK, Lucas RM, Banks E, Ponsonby AL. Variability in vitamin $D$ assays impairs clinical assessment of vitamin $D$ status. Intern Med J 2012;42:43-50. https://doi.org/10.1111/ j.1445-5994.2011.02471.x

6. Sempos CT, Vesper HW, Phinney KW, Thienpont LM, Coates $P M$. Vitamin D status as an international issue: national surveys and the problem of standardization. Scand J Clin Lab Invest Suppl 2012;243:32-40.

7. Clinical Laboratory Standards Institute. Evaluation of precision performance of quantitative measurement methods; approved guideline. CLSI document EP05-A2. CLSI: Wayne, $P A, 2004$.

8. Clinical Laboratory Standards Institute. Protocols or Determination of Limits of Detection and Limits of Quantitation; approved guideline. CLSI document EP17-A2. CLSI: Wayne, PA, 2012.

9. Clinical Laboratory Standards Institute. Evaluation of the Linearity of Quantitative Measurement Procedures: A Statistical Approach; approved guideline. CLSI document EP06-A. CLSI: Wayne, PA, 2003.

10. Clinical Laboratory Standards Institute. Interference Testing in Clinical Chemistry; approved guideline. CLSI document EP07-A2. CLSI: Wayne, PA, 2005.

11. Chen $Y$, Kinney L, Soldin SJ. Performance evaluation of Siemens ADVIA Centaur enhanced estradiol assay and a split sample comparison with liquid chromatography-tandem
Based on the present criteria, all immunoassays can be used in routine 25(OH)D measurements, still fairly diagnosing the patients' status. Recent standardization attempts do not seem to contribute too much to clinical diagnosis. At least a clinical laboratory must be aware of its method to avoid the misinterpretation of results.

\section{Potential conflict of interest}

None declared.

mass spectrometry. Clin Biochem 2012;45:811-5. https:// doi.org/10.1016/j.clinbiochem.2012.02.016

12. Chen $Y$, Yazdanpanah $M$, Hoffman BR, Diamandis EP, Wong $P Y$. Rapid determination of serum testosterone by liquid chromatography-isotope dilution tandem mass spectrometry and a split sample comparison with three automated immunoassays. Clin Biochem 2009;42:484-90. https://doi. org/10.1016/j.clinbiochem.2008.11.009

13. Clinical Laboratory Standards Institute. Method Comparison and Bias Estimation Using Patient Samples; approved guideline. CLSI document EP09-A2-IR. CLSI: Wayne, PA, 2010. (Interim Revision).

14. McHugh ML. Interrater reliability: the kappa statistic. Biochem Med (Zagreb) 2012;22:276-82. https://doi. org/10.11613/BM.2012.031

15. Janssen MJ, Wielders JP, Bekker CC, Boesten LS, Buijs MM, Heijboer AC, et al. Multicenter comparison study of $\mathrm{Cu}$ rrent methods to measure 25-hydroxyvitamin $D$ in serum. Steroids 2012;77:1366-72. https://doi.org/10.1016/j.steroids.2012.07.013

16. Farrell CJ, Martin S, McWhinney B, Straub I, Williams $P$, Herrmann M. State-of-the-art vitamin $D$ assays: $a$ comparison of automated immunoassays with liquid chromatography-tandem mass spectrometry methods. Clin Chem 2012;58:531-42. https://doi.org/10.1373/ clinchem.2011.172155

17. Binkley N, Krueger D, Cowgill CS, Plum L, Lake E, Hansen $K E$, et al. Assay variation confounds the diagnosis of hypovitaminosis D: a call for standardization. J Clin Endocrinol Metab 2004;89:3152-7. https://doi.org/10.1210/jc.2003031979

18. Enko D, Fridrich L, Rezanka E, Stolba R, Ernst J, Wendler I, et al. 25-hydroxy-vitamin $D$ status: limitations in comparison and clinical interpretation of serum-levels across different assay methods. Clin Lab 2014;60:1541-50. https://doi. org/10.7754/Clin.Lab.2014.131114

19. Enko D, Kriegshäuser G, Stolba R, Worf E, Halwachs-Baumann $G$. Method evaluation study of a new generation of vitamin D assays. Biochem Med (Zagreb) 2015;25:203-12. https://doi.org/10.11613/BM.2015.020 
20. Simpson CA, Cusano AM, Bihuniak J, Walker J, Insogna KL. Effect of $25(\mathrm{OH})$ vitamin $D$ reference method procedure (RMP) alignment on clinical measurements obtained with the IDS-iSYS chemiluminescent-based automated analyzer. J Steroid Biochem Mol Biol 2015;148:41-6. https://doi. org/10.1016/j.jsbmb.2014.09.013

21. U.S. Department of Health and Human Services, Food and Drug Administration (FDA), Center for Drug Evaluation and Research (CDER), Center for Veterinary Medicine (CVM). Guidance for Industry - Bioanalytical Method Validation - Revision 1. September 2013. Available at: http://www.fda.gov/ downloads/Drugs/GuidanceComplianceRegulatorylnformation/Guidances/UCM368107.pdf. Accessed December 10th 2016.

22. Fraser CG, ed. Biological variation: from principles to practice. Washington (DC): AACC Press; 2001.

23. Viljoen $A$, Singh DK, Farrington K, Twomey PJ. Analytical quality goals for 25-vitamin $D$ based on biological variation. J Clin Lab Anal 2011;25:130-3. https://doi.org/10.1002/ jcla.20446

24. Stöckl D, Sluss PM, Thienpont LM. Specifications for trueness and precision of a reference measurement system for serum/plasma 25-hydroxyvitamin D analysis. Clin Chim Acta 2009;408:8-25. https://doi.org/10.1016/j.cca.2009.06.027
25. Wyness SP, Straseski JA. Performance characteristics of six automated 25-hydroxyvitamin D assays: Mind your 3s and 2s. Clin Biochem 2015;48:1089-96. https://doi.org/10.1016/j. clinbiochem.2015.08.005

26. Binkley \&, Krueger DC, Morgan S, Wiebe D. Current status of clinical 25-hydroxyvitamin $D$ measurement: An assessment of between-laboratory agreement Clin Chim Acta 2010;411:1976-82. https://doi.org/10.1016/j. cca.2010.08.018

27. Carter GD. Accuracy of 25-hydroxyvitamin D assays: confronting the issues. Curr Drug Targets 2011;12:19-28. https:// doi.org/10.2174/138945011793591608

28. Kushnir MM, Ray JA, Rockwood AL, Roberts WL, Laulu $S L$, Whittington JE, et al. Rapid analysis of 25-hydroxyvitamin D2 and D3 by liquid chromatography-tandem mass spectrometry and association of vitamin $D$ and parathyroid hormone concentrations in healthy adults. Am J Clin Pathol 2010;134:148-56. https://doi.org/10.1309/ AJCPPIA7DFBT4GKS

29. Singh RJ, Taylor RL, Reddy GS, Grebe SK. C-3 epimers can account for a significant proportion of total circulating 25-hydroxyvitamin $D$ in infants, complicating accurate measurement and interpretation of vitamin D status. J Clin Endocrinol Metab 2006;91:3055-61. https://doi.org/10.1210/ jc.2006-0710 